\title{
Multi-Disciplinary Design Optimization under Uncertainty for Thermal Protection System Applications
}

\author{
Jiulong Sun, Geng Zhang, \\ Michigan Engineering Services, LLC \\ 3916 Trade Cnter Drive, Ann Arbor, MI \\ Nick Vlahopoulos (corresponding author) \\ University of Michigan \\ Dept. of Naval Architecture and Marine Engineering / Mechanical Engineering \\ 2660 Draper Road, Ann Arbor, MI \\ Sang-Bum Hong, \\ Cummins \\ MC 50182 \\ 1900 McKinley Avenue \\ Columbus, IN 47201
}

\begin{abstract}
This paper presents an approach for performing Multi-disciplinary Design Optimization under Uncertainty (MDO-U) and an application for minimizing the thickness of the Thermal Protection System (TPS) for an entry vehicle configuration. Uncertainties due to material variability and the operating environment are considered. The value of considering uncertainty during the optimization process is demonstrated by comparing the performance of two optimal configurations, one identified through a deterministic approach and the other by considering uncertainty. Been able to account for uncertainty in engineering simulations and in product design is both important and challenging. In particular, during design optimization the performance of the optimized design typically lays closely to the boundaries of some of the constraints. Since variability alters the actual performance of a system, the optimal design can exhibit performance in the infeasible domain when the impact of the uncertainty has not been accounted properly during the optimization.
\end{abstract}

\section{Introduction}

The physical difficulty of designing entry vehicles originates from the large degree of coupling between the various disciplines involved in the design [1-3]. The disciplines which can be accounted for and integrated during the design are: trajectory optimization [4-6], guidance, navigation, and control (GN\&C) technology $[7,8]$, aerodynamics and aerothermodynamics [911], thermal-structural analysis [12-14], and thermal protection system (TPS) development [1519]. Efforts have been made in developing a collaborative or a multidisciplinary optimization process that considers some of the disciplines of interest during an integrated design [20-23]. However, none of these efforts considers how uncertainty in the atmospheric conditions, in the entry parameters of the vehicle, in the condition of the vehicle during entry, and in the performance of the TPS will influence the design and provide a risk assessment. The work presented in this paper demonstrates that it is feasible to utilize a MDO-U tool for entry vehicle design, and that the new information is insightful and meaningful. The new MDO-U development can take into account how uncertainties influence the modeling results of each 
discipline, and how uncertainties influence the interaction between disciplines and the optimal solution. A Multi-discipline Design Optimization (MDO) method available in the literature [24 -28] is utilized for developing a general purpose MDO environment. The uncertainty from the variability of the random variables and other random parameters is introduced through an approximate but computationally efficient first - order reliability method [29, 30]. A brief theoretical overview of the methods employed in this work is presented first. A case study where MDO-U analysis is performed for a configuration of the HL-20 vehicle (Figure 1) under a LEO consideration is presented.

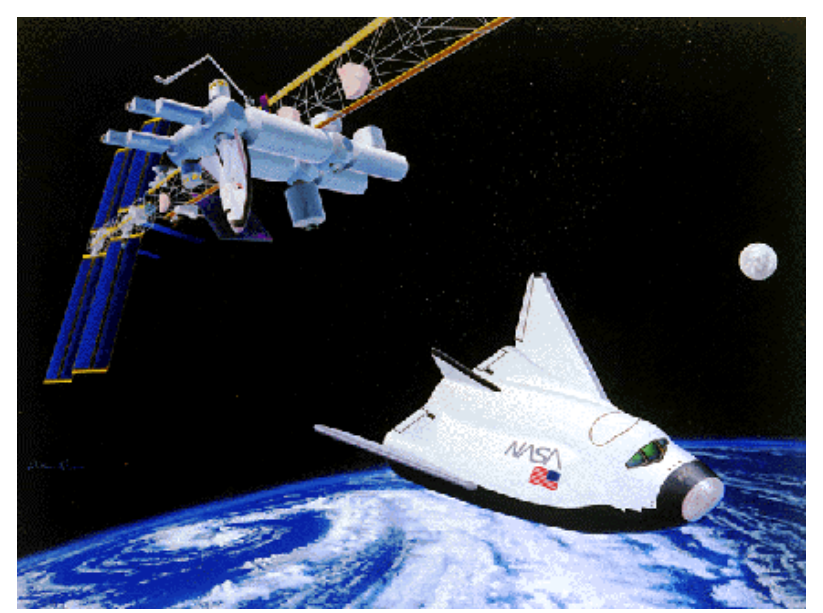

Figure 1. HL20 Vehicle used in the MDO-U case study

(figure from http://planet.esuhsd.org/resources/images/artist_concept_pls.html)

Three different types of TPS (AFRSI, PICA, and SIRCA) are considered to be applied on different sections of the HL-20 (Figure 2). The trajectory code Traj [31] developed by NASA Ames is employed for the trajectory computations. Aerothermal information is computed at each point of the trajectory from flight characteristics computed by Traj (mach number, angle of attack, and dynamic pressure) and an aerothermal database provided by NASA Ames and created using the CBAERO package $[32,33]$. The CBAERO code has been utilized in the past for designing the trajectory and the thermal protection system for reusable launch vehicles [34]. For the HL-20 configuration the CBAERO code has been validated through comparison to test data for the integrated lift, drag, and moment coefficients for subsonic through supersonic Mach numbers [32]. Thermal analyses are performed for each one of the three TPS systems using finite elements. The thermal loads are determined from the trajectory results and the aerothermal database. The trajectory computations provide information about the Mach number, the angle of attack, and the dynamic pressure at each step of the time history. Based on the Mach number, the angle of attack, and the dynamic pressure the aerothermal database provides information for the corresponding heat flux at different locations of interest on the vehicle. In this manner the heat flux time histories are generated for the different locations where thermal TPS analyses are performed. Properties from the NASA Ames TPS database TPSX [35] are employed in the thermal finite element models for the three TPS systems.

Both a MDO analysis without uncertainty, and a MDO-U (with uncertainty) are performed. Uncertainties are considered in the atmospheric properties and the heat transfer characteristics of the TPS. The uncertainties in the atmospheric properties introduce variability in the heat load applied on the vehicle, and the uncertainties in the heat transfer properties of the TPS alter its 
thermal performance. Thus, it is critical to consider these variations during the optimization in order to ensure that the final design will satisfy the safety and the performance constraints even in the presence of uncertainty. The results from the MDO and the MDO-U of the HL20 are discussed in this paper.

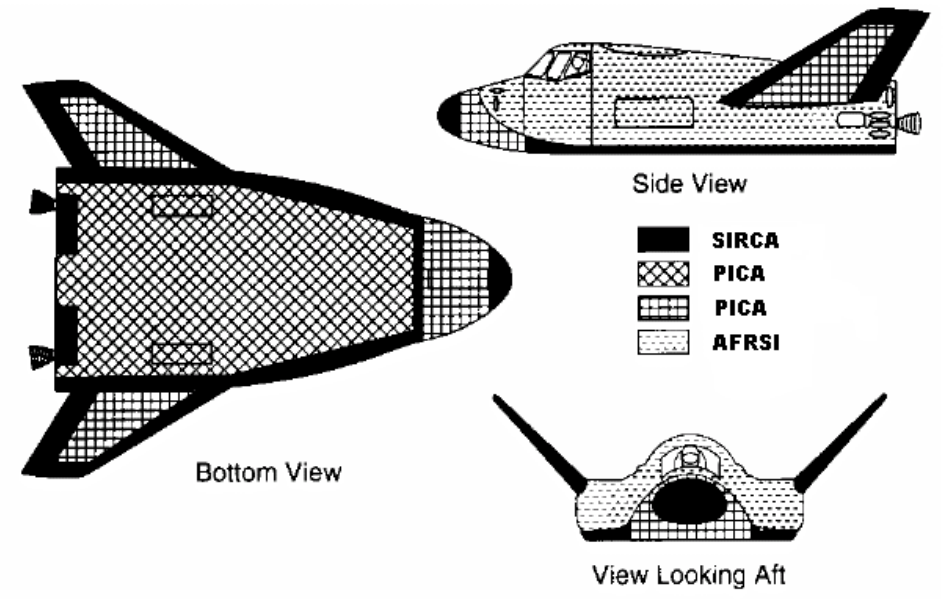

Figure 2. Distribution of TPS material over the surface of the HL-20 considered in the case study

\section{Overview of Theories for MDO and for Including Uncertainty in Optimization II.1. Theory Overview for $\mathrm{MDO}$}

Work presented in [24 - 28] provides the theoretical foundation for developing the MDO environment. Figure 3 presents a general flow chart of the MDO process. At the top level of the optimization process lays the optimization statement which defines the overall objective for the entry vehicle. The top level optimization, tries to make the vehicle's performance represented by the $P_{v}$ response to match the targeted performance defined as $T_{v}$ by the user. The vehicle evaluation model takes into account the variables describing the performance of the vehicle $\left(P_{D_{1}}, \ldots, P_{D_{N}}\right)$ in each one of the $\mathrm{N}$ disciplines $\left(D_{1}, \ldots, D_{N}\right)$ which are considered. The vehicle evaluation model also takes into account the variables $X_{v}$ which define the design characteristics of the vehicle. The vehicle evaluation model constitutes a mathematical statement which assesses how the design variables and the performance from each discipline are combined in order to evaluate the overall performance. In this manner, when the expectations for the performance from each discipline are defined, the corresponding impact on the overall objective function is identified. Within each iteration of the top level vehicle design optimization process, a separate optimization problem is solved for each discipline. The linking design variables $l_{D_{i}}^{T}$ are passed from the top "T", level optimization, to the optimization problem at each discipline level. The performance of each discipline desired by the top optimization problem is also defined as $P_{D_{i}}^{T}$.

At each discipline level a design optimization problem is solved using the appropriate analysis model. Within each iteration of the discipline level optimization problem, the design variables $X_{D_{i}}$ and the linking variables $l_{D_{i}}$ are provided to the simulation model. The predicted performance $P_{D_{i}}$ is returned from the simulation model. Once the discipline level optimization analysis is completed and the optimal solution is reached, the updated "U" values for the linking 
variables $l_{D_{i}}^{U}$ and the updated performance $P_{D_{i}}^{U}$ are returned to the top level optimization problem. Then, the next iteration in the top level optimization problem is performed.

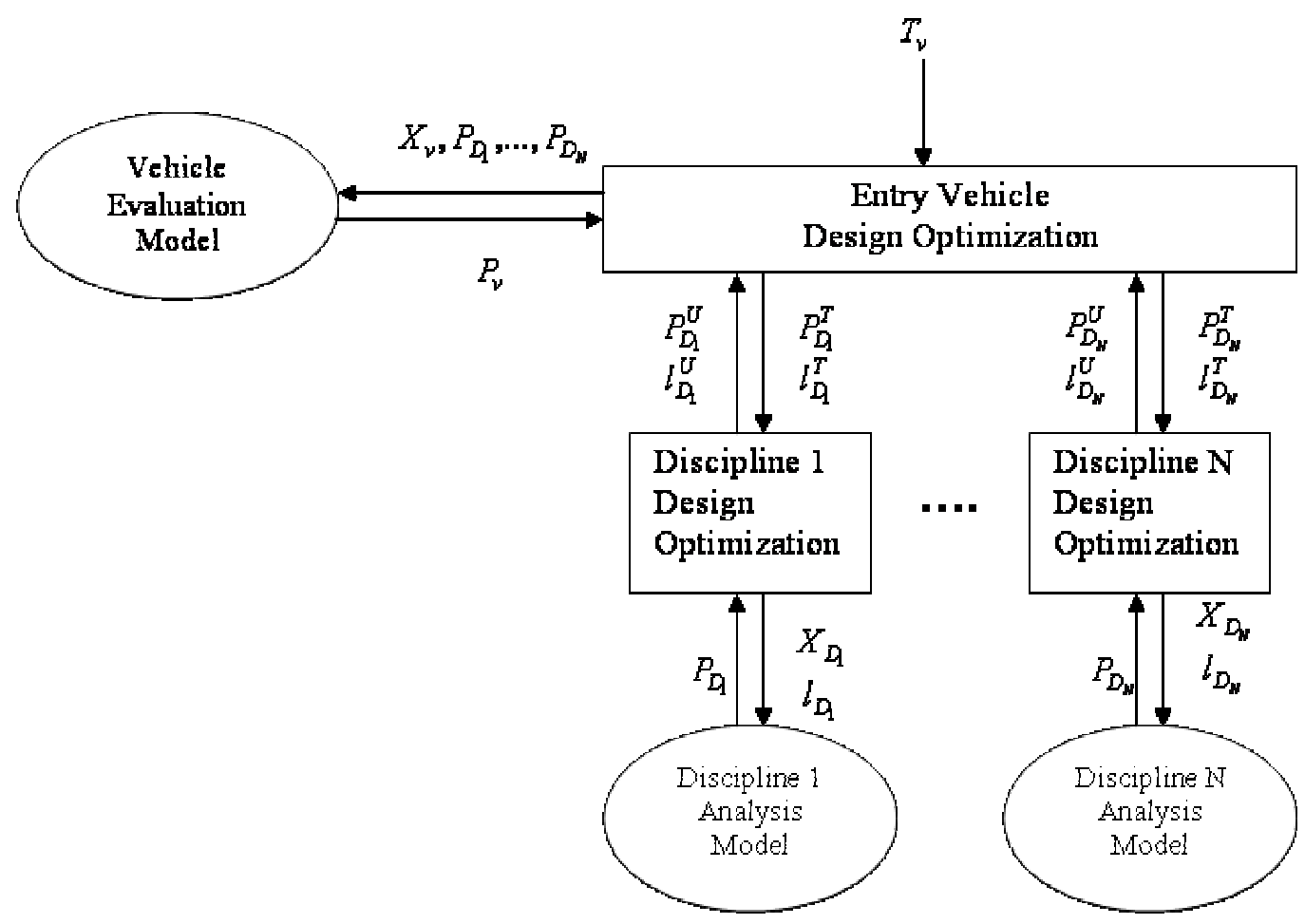

Figure 3. Flow chart for MDO process

The mathematical statement for the top level optimization problem is:

$$
\min _{X_{v}, P_{D_{i}}, l_{D_{i}}}\left\|P_{v}-T_{v}\right\|+\varepsilon_{v}^{P}+\varepsilon_{v}^{l}
$$

subject to: $\sum_{i=1}^{N}\left\|P_{D_{i}}-P_{D_{i}}^{U}\right\| \leq \varepsilon_{v}^{P}, \sum_{i=1}^{N}\left\|l_{D_{i}}-l_{D_{i}}^{U}\right\| \leq \varepsilon_{v}^{l}, g_{v}\left(P_{V_{i}}, X_{v}, l_{D_{i}}\right) \leq 0, h_{v}\left(P_{V_{i}}, X_{v}, l_{D_{i}}\right)=0$

where $g_{v}$ presents the inequality constraints and $h_{v}$ presents the equality constraints of the top level design optimization problem. The mathematical statement for each discipline level design optimization problem is:

$$
\min _{X_{D_{i}}, l_{D_{i}}}\left\|P_{D_{i}}-P_{D_{i}}^{T}\right\|
$$

subject to: $g_{D_{i}}\left(P_{D_{i}}, X_{D_{i}}, l_{D_{i}}\right) \leq 0, h_{D_{i}}\left(P_{D_{i}}, X_{D_{i}}, l_{D_{i}}\right)=0$ 
Introducing constraints on the change of the linking variables and on the change of the performance variables at each top level iteration, and augmenting the objective function of the top level optimization by adding to the objective function the bounds of the extra constraints, limits the amount of changes introduced by each discipline within each top level iteration. In this manner extreme changes in the overall system are avoided and the design variables are adjusted gradually towards an improved configuration.

The overall design optimization objective is achieved by letting the top level optimization (which is based on the performance of each discipline) to exchange information with each discipline in a very systematic manner. Different disciplines can share the same variables since elements of the $l_{D_{i}}$ groups of linking variables can be the same for different groups. Thus, the inter-relationships between disciplines are accounted, while retaining a very structured and efficient manner for optimizing the design.

\section{II.2. Introducing Uncertainty in the MDO system}

The introduction of uncertainty in the MDO simulations is based on a first order reliability approximation which has been presented in the literature [29, 30]. According to this approach instead of evaluating a probabilistic constraint which requires time consuming computations:

$P(G(\mathbf{d}, \mathbf{X}, \mathbf{p}) \geq 0) \geq R$

where $G$ is the constraint function, $\mathbf{d}$ is the vector of the deterministic design variables, $\mathbf{X}$ is the vector of the random design variables, $\mathbf{p}$ is the vector of the random parameters, and $R$ is the desired reliability level for the constraint function to satisfy the condition of being greater or equal to zero; instead, the probabilistic constraint is stated as a deterministic one:

$G(\mathbf{d}, \mathbf{X}, \mathbf{p}) \geq 0$

where

$$
\begin{aligned}
& \mathbf{X}=\boldsymbol{\mu}_{\mathbf{X}}-\boldsymbol{\sigma} * \beta_{\mathrm{t}} * \boldsymbol{\alpha} \\
& \mathbf{p}=\boldsymbol{\mu}_{\mathbf{p}}-\boldsymbol{\sigma} * \beta_{\mathrm{t}} * \boldsymbol{\alpha} \\
& \boldsymbol{\alpha}=\boldsymbol{\sigma} * \nabla G_{\mathbf{X}, \mathbf{p}}(\mathbf{d}, \mathbf{X}, \mathbf{p}) /\left\|\boldsymbol{\sigma} * \nabla G_{\mathbf{X}, \mathbf{p}}(\mathbf{d}, \mathbf{X}, \mathbf{p})\right\|
\end{aligned}
$$

Equation (4) evaluates the constraints which includes the random variables and /or random parameters as a deterministic constraint. It uses a first order reliability approximation for evaluating the constraints at a point assessed by Equation (5), thus introducing a safety margin in each constraint based on the desired reliability level. This is an approximate but extremely efficient manner for introducing uncertainty in an optimization process. In this work Equation (5) is utilized for identifying the location where constraints and functions which depend on random design variables and random parameters should be evaluated. When objective functions depend on random design variables and on random parameters, then the mean values of the random quantities are utilized for evaluating the objective functions. 


\section{Analysis of a HL20 configuration}

A MDO without uncertainty and a MDO-U analysis are performed for a configuration of the HL20 in order to demonstrate the new capabilities developed in this work and the importance of including uncertainties in the optimization process. The simulations involved in the optimizations included, trajectory computations using the Traj code provided by NASA Ames [31], aerodynamic analysis (included within the Traj code), aerothermal analysis through the use of aerothermal databases developed by CBAERO [32,33], and thermal analyses for the three different TPS configurations (AFRSI, PICA, and SIRCA) which are considered placed at different sections of the vehicle.

The flow chart of the optimization analyses is presented in Figure 4. In this case in the top level optimization the objective is to minimize the total mass of the TPS material applied on the three different sections of the vehicle (Figure 2). Nine design variables are considered in the optimization process which are the same nine design variables used by the four discipline level optimization analyses. Thirteen constraints are also defined for the top level optimization according to Equations (1) (nine for each one of the nine design variables shared with the discipline level optimizations, and four for each one of the four objective functions of the discipline level optimizations). The four discipline level optimizations which are considered are: (1) trajectory optimization for the landing location of the vehicle; (2) AFRSI TPS optimization; (3) PICA TPS optimization; (4) SIRCA TPS optimization.

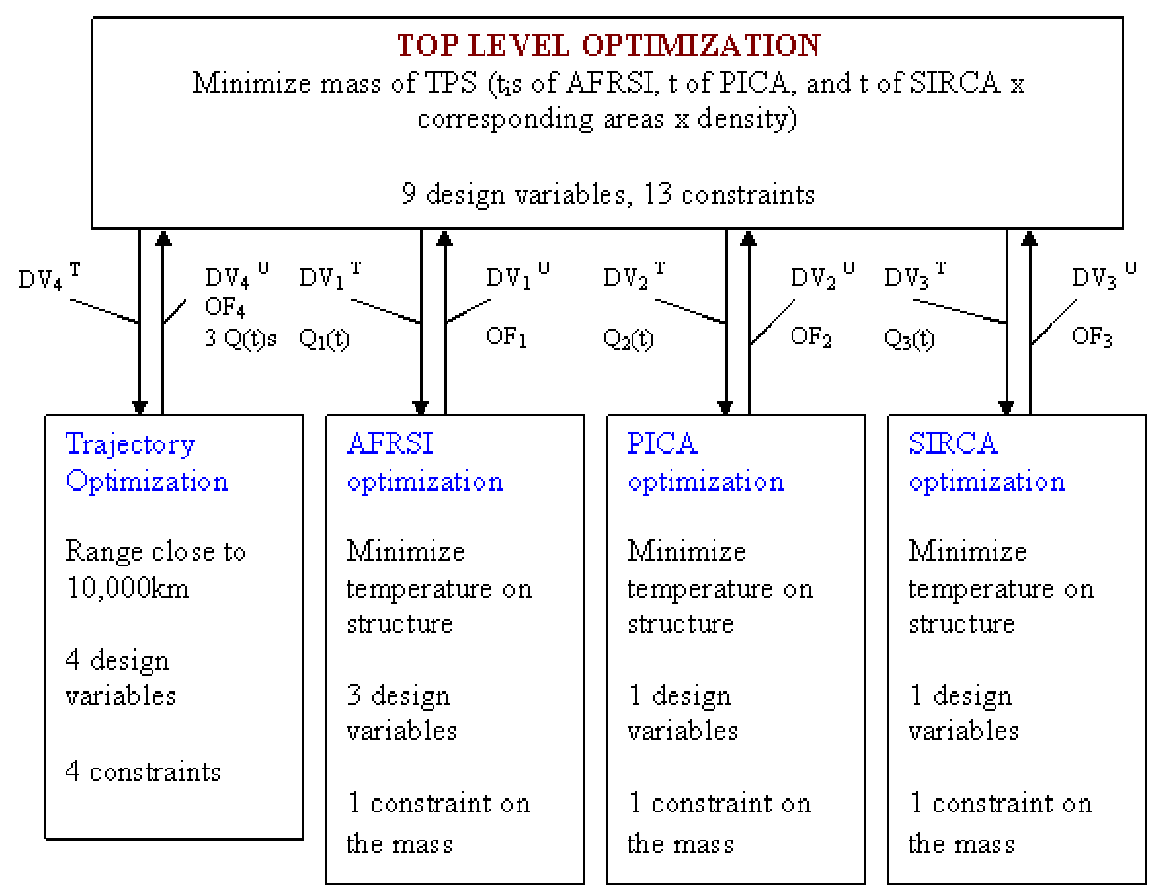

Figure 4. Flow chart of Optimization Process for HL20 case study 
The Traj code [31] is employed for the trajectory calculations and for the aerodynamic analysis. In addition to the trajectory computations it evaluates aerodynamic type of information (mach number, dynamic pressure, angle of attack) which when combined with the aerothermal database created by the CBAERO code allows to evaluate the heat flux time histories at different positions of the vehicle. Finite element models are developed for the AFRSI TPS (Figure 5) and the PICA and SIRCA TPS [ 15] (Figure 6).

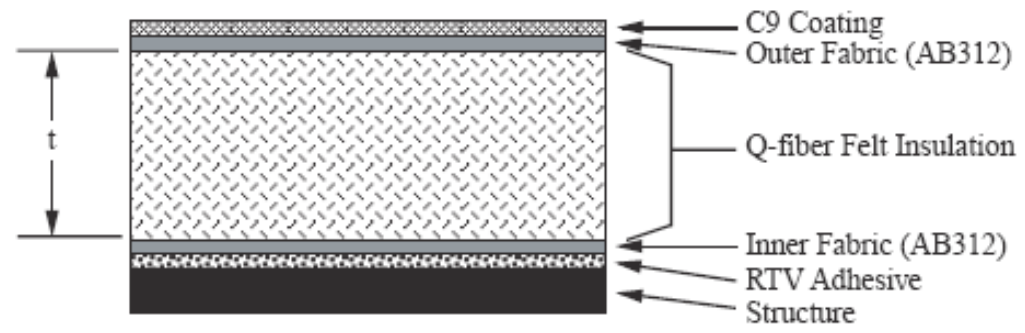

Figure 5. AFRSI TPS configuration from [19]

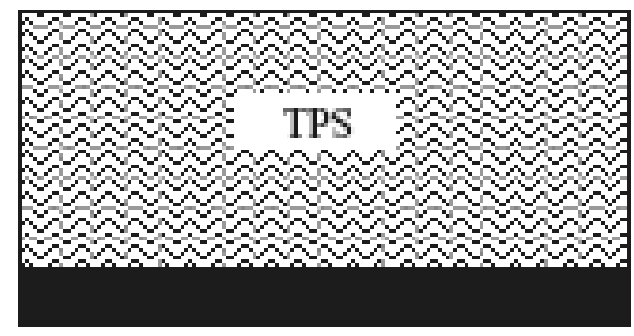

Figure 6. PICA and SIRCA TPS configurations

In the first discipline level optimization the trajectory is optimized. The objective function reflects the requirement of making the range as close to $10,000 \mathrm{~km}$ as possible. The conditions at the entry point comprise the design variables for this discipline level optimization. Specifically, the initial velocity, the initial angle of attack, the initial entry angle, and the initial bank angle comprise the four design variables. Constraints are also imposed on the maximum values exhibited by the dynamic pressure, the heat flux, the end altitude, and the heat load. This set up is not the only possible manner for performing the optimization. It is just used as a representative approach in this case study. In the discipline level thermal optimizations for the three TPS, each objective function targets to minimize the maximum temperature encountered on the structural skin of the TPS. For the AFRSI optimization, the thicknesses of three layers (outer fabric, Q-fiber felt insulation, and inner fabric) comprise the three design variables. Constraints are imposed on the maximum mass of the AFRSI TPS and a convergence constraint for the thermal analysis is employed in order to ensure that convergence has been achieved. The other two discipline level optimizations (for the PICA and the SIRCA) are set up in a similar manner. The only difference is that for the PICA and the SIRCA, only one design variable is considered in each corresponding discipline level optimization (the thickness of the TPS Figure 6). Three heat fluxes are determined from the trajectory calculations and from the aerothermal database, one for each TPS configuration. The heat flux at a point at the top of the canopy is used in the 
AFRSI thermal computations. The heat flux in the middle bottom location of the vehicle is used for the PICA thermal analysis. Finally, the maximum heat flux between a point at the nose and a point at the leading edge of the wing provides the thermal load for the SIRCA thermal analysis.

\section{III.1. MDO analysis without Uncertainty}

The results from the MDO without uncertainty are discussed first. An initial setting for the four variables of the trajectory analysis is considered (initial velocity $=7.8976 \mathrm{~km} / \mathrm{sec}$, initial gamma angle $=-1.3^{\circ}$, initial angle of attack $=28^{\circ}$, and initial bank angle $=60^{\circ}$ ). For this initial setting, the thicknesses assigned to each one of the three TPS systems are optimized in order to achieve a maximum temperature on the structural skin of approximately $460^{\circ} \mathrm{K}$. This is a configuration that reduces the thickness of the TPS as much as possible for the initial trajectory settings. Thus, at the starting point the three thickness design variables for the AFRSI are $(5 \mathrm{~mm}$, $20 \mathrm{~mm}$, and $6 \mathrm{~mm})$. The thickness for the PICA is $3.2 \mathrm{~cm}$, and the thickness for the SIRCA is $6 \mathrm{~cm}$. The total initial mass for the TPS at the starting point of the MDO is $303 \mathrm{~kg}$. These initial values and the corresponding values for the optimum configuration identified from the MDO without uncertainty are summarized in Table 1.

\begin{tabular}{|l|c|c|}
\hline & \multicolumn{1}{|c|}{$\begin{array}{c}\text { Initial point for } \\
\text { optimization }\end{array}$} & $\begin{array}{c}\text { Optimized } \\
\text { configuration }\end{array}$ \\
\hline initial velocity $(\mathrm{km})$ & 7.8976 & 7.9148 \\
\hline initial angle of attack & 28 & 33.58 \\
\hline initial entry angle & -1.3 & -1.32 \\
\hline initial bank angle & 60 & 57.19 \\
\hline $\begin{array}{l}\text { thickness of outer fabric in AFRSI } \\
\text { (mm) }\end{array}$ & 5 & 4 \\
\hline thickness of Q-fiber in AFRSI $(\mathrm{mm})$ & 20 & 15.89 \\
\hline $\begin{array}{l}\text { thickness of inner fabric in AFRSI } \\
\text { (mm) }\end{array}$ & 6 & 4.8 \\
\hline thickness of PICA $(\mathrm{cm})$ & 3.2 & 2.53 \\
\hline thickness of SIRCA $(\mathrm{cm})$ & 6 & 4.9 \\
\hline total mass of TPS $(\mathrm{kg})$ & 303 & 242 \\
\hline
\end{tabular}

Table 1. Initial and final optimum from MDO without uncertainty

The heat flux associated with the SIRCA material is the highest one of the three heat loads which are used for the design of each TPS, therefore the results associated with the optimal configuration for the SIRCA will be discussed in more detail, as representative of the discipline level optimizations. The heat flux load for the starting point of the optimization, and the heat flux for the optimum configuration after the MDO are presented in Figure 7 . The corresponding temperature histories on the surface of the structure are presented in Figure 8. It can be observed that for the optimum configuration, the heat load has been reduced due to the modifications introduced in the trajectory profile and thus it is possible to achieve an even lower temperature on the surface of the structure even with a reduced thickness for the TPS material. These results demonstrate how the MDO method utilized in this work operates for entry vehicle applications, and how multiple objectives can be accomplished simultaneously. 


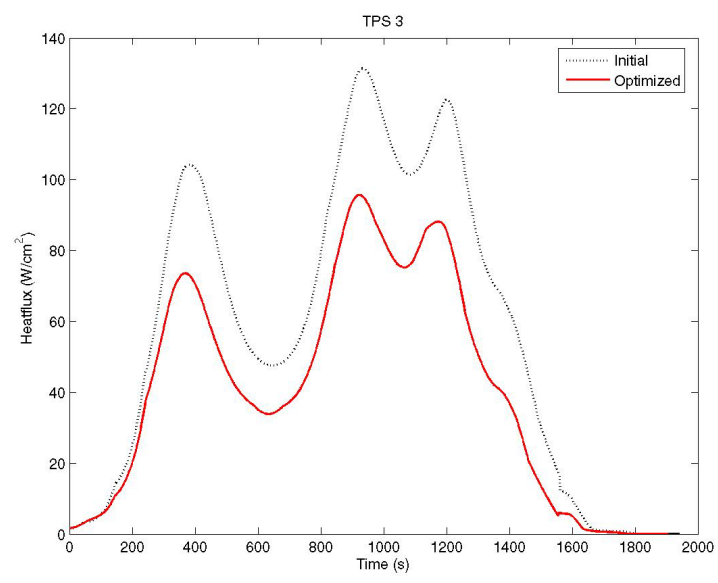

Figure 7. Heat flux imposed on the SIRCA TPS for the starting point of the MDO and for the optimized configuration

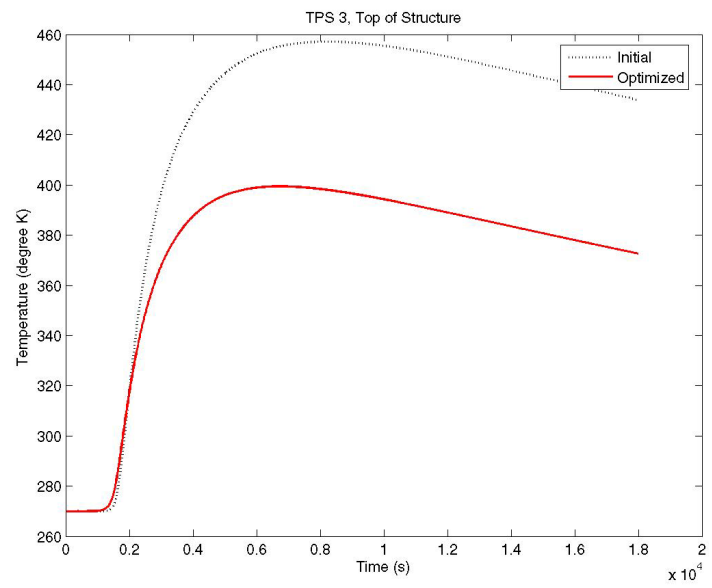

Figure 8. Temperature on the top of the structural skin of the SIRCA TPS for the starting point of the MDO and the optimized configuration

\section{III.2 MDO Analysis under Uncertainty}

Uncertainties are introduced in the MDO computations by considering uncertainties in the atmospheric properties and in the thermal properties of the TPS material. The atmospheric properties are considered to influence the convective heating model employed in the Traj code [35]. Specifically, the uncertainty is introduced in the Tauber model [36] by considering uncertainty for the value of the exponent $\eta$.

$q_{c n v}=C_{c v n} v_{\text {inf }}^{\eta} \sqrt{\frac{\rho_{\text {inf }}}{r_{\text {nose }}}}\left[1-\frac{h_{\text {wall }}}{h_{\text {edge }}}\right]$

Similarly uncertainty is considered in the specific heat associated with the material of each one of the three TPS configurations. The specific heat values for the TPS materials and the exponent $\eta$ comprise the random parameters. A normal distribution is considered for each one of the 
random parameters with a standard deviation equal to $10 \%$ of the mean value of each random parameter. The MDO-U analysis is performed under a requirement for a $98 \%$ reliability level. The first order reliability method employed in this effort requires evaluation of the gradients of the constraint functions and of any other functions which depend on the random parameters, according to Equation (5). In this manner the appropriate locations where the constraints should be evaluated are identified in order to ensure that the constraints are satisfied with a $98 \%$ reliability level even in the presence of uncertainty. In this case study the two constraint functions associated with the heat load and the heat flux in the trajectory optimization discipline depend on the random parameters. In addition, the heat flux time histories which are evaluated from the trajectory simulation and applied as excitation in the three other discipline level optimizations are functions which depend on parameters with uncertainty. Finally, the constraints on the mass of each TPS discipline level optimization depend on the specific heat of the material since different thickness can be required for keeping the temperature of the structure below an acceptable limit when uncertainty exists in the specific heat.

The entry conditions for the starting point of the optimization are the same for the trajectory calculations as in the case of the MDO without uncertainty (initial velocity $=7.8976 \mathrm{~km} / \mathrm{sec}$, initial gamma angle $=-1.3^{\circ}$, initial angle of attack $=28^{\circ}$, and initial bank angle $=60^{\circ}$ ). For this initial setting, the thicknesses assigned to each one of the three TPS systems are optimized again as in the MDO case in order to achieve a maximum temperature on the structural skin of approximately $460^{\circ} \mathrm{K}$, but this time in the presence of uncertainty and under the requirement of a $98 \%$ reliability level. The information associated with the initial point of the MDO-U and with the optimized point of the MDO-U are summarized in Table 2.

\begin{tabular}{|l|c|c|}
\hline & \multicolumn{1}{|c|}{$\begin{array}{c}\text { Initial point for } \\
\text { optimization }\end{array}$} & $\begin{array}{c}\text { Optimized } \\
\text { configuration }\end{array}$ \\
\hline initial velocity $(\mathrm{km})$ & 7.8976 & 7.8454 \\
\hline initial angle of attack & 28 & 33.38 \\
\hline initial entry angle & -1.3 & -0.94 \\
\hline initial bank angle & 60 & 55.26 \\
\hline $\begin{array}{l}\text { thickness of outer fabric in AFRSI } \\
(\mathrm{mm})\end{array}$ & 5 & 3.35 \\
\hline thickness of Q-fiber in AFRSI $(\mathrm{mm})$ & 20 & 19.14 \\
\hline $\begin{array}{l}\text { thickness of inner fabric in AFRSI } \\
\text { (mm) }\end{array}$ & 6 & 5.1 \\
\hline thickness of PICA $(\mathrm{cm})$ & 4.2 & 3.35 \\
\hline thickness of SIRCA $(\mathrm{cm})$ & 9.4 & 7.6 \\
\hline total mass of TPS $(\mathrm{kg})$ & 366 & 302 \\
\hline
\end{tabular}

Table 2. Initial and final optimum from MDO-U

When uncertainty is present in the simulations, the starting point of the optimization exhibits a higher mass compared to the starting point of the MDO without uncertainty. This increase in the mass is required in order to unsure a maximum temperature on the structural skin of the TPS at approximately $460^{\circ} \mathrm{K}$ even in the presence of uncertainty. Since the heat flux associated with the SIRCA material is the highest one of the three heat loads which are used for the design of each TPS, results for the SIRCA are discussed in more detail, similar to the discussion for the MDO 
without uncertainty. The heat flux load for the starting point of the optimization, and the heat flux for the optimum configuration after the MDO are presented in Figure 9. These heat loads are higher than the ones encountered in the MDO without uncertainty (Figure 7) because they represent the $98 \%$ reliability level. It can be observed that for the optimum configuration, the heat load has been reduced due to the modifications introduced in the trajectory profile and thus it is possible to achieve an even lower temperature on the surface of the structure with a reduced thickness for the TPS material even in the presence of uncertainty. Finally the temperature on the outer part of the structural skin of the SRICA is presented in Figure 10. It can be observed that the MDO-U led to a configuration which exhibits lower weight for the TPS and yet good thermal protection for the structure even in the presence of variability due to uncertainties.

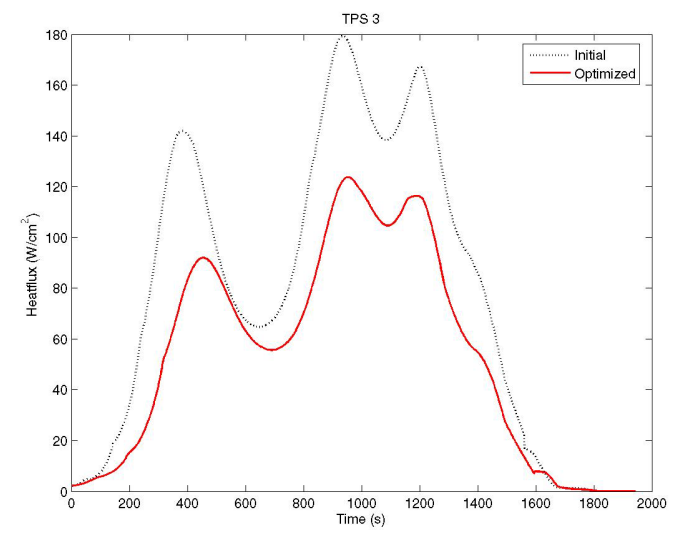

Figure 9. Heat flux imposed on the SIRCA TPS for the starting point of the MDO-U and for the optimized configuration corresponding to the $98 \%$ reliability level

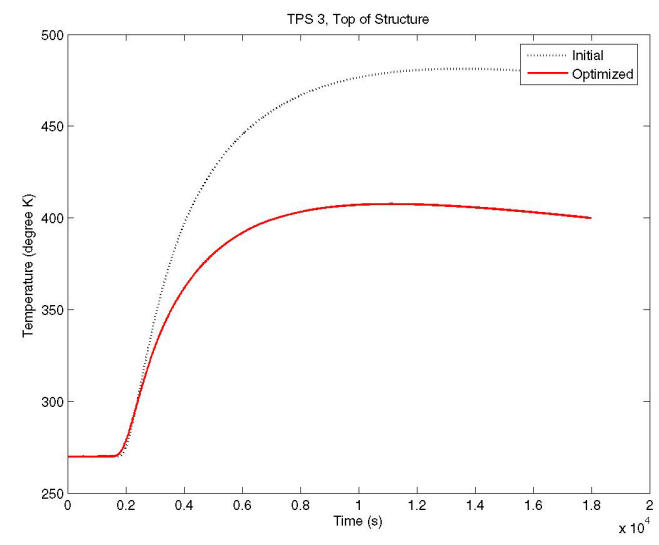

Figure 10. Temperature on the top of the structural skin of the SIRCA TPS for the starting point of the MDO-U and the optimized configuration corresponding to a $98 \%$ reliability level

\section{III.3. Importance of Uncertainty in the $\mathrm{MDO}$ simulations}

In order to demonstrate the importance of accounting for uncertainty when performing the optimization, a comparison is made between the performances of the TPS which was identified by the MDO process without uncertainty and the configuration identified by the MDO-U analysis. A safety factor is applied on the optimum configuration identified by the former process by increasing the thickness of the TPS and making the total mass equal to the mass of the 
optimal configuration identified by the MDO-U analysis. Thus, both configurations have the same weight for the TPS, but in the second case the configuration is identified through the MDO-U process which considers the effects of uncertainty. In this case the comparison is made by considering uncertainties in the atmosphere and in the thermal properties of the TPS, as it is done during the MDO-U computations. The results for the temperature on the top part of the structural skin of the SRICA are presented in Figure 11. It can be observed that the configuration which corresponds to the optimal point without uncertainty, although it exhibits the same mass with the optimized configuration of the MDO-U, it exhibits inferior performance in the presence of uncertainty. The reason for this relative behavior is that when uncertainty is considered, both the adjustments in the trajectory and the changes in the thicknesses of the TPS reflect the influence of the uncertainty, while the introduction of the safety factors are only applied on the thickness and do not alter the optimal trajectory. Thus, the importance of the new MDO-U process is demonstrated.

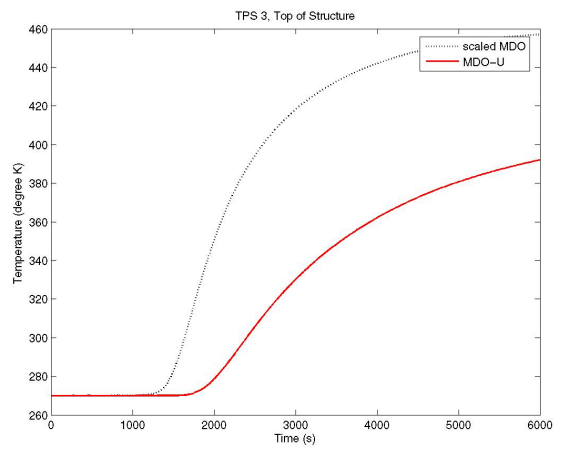

Figure 16. Temperature on the top of the structural skin of the SIRCA TPS for the starting point of the MDO without uncertainty and for the optimized configuration from the MDO-U corresponding to a $98 \%$ reliability level

\section{Summary}

In this paper an approach is presented for including uncertainty in MDO simulations for thermal protection system applications. Trajectory, aerodynamic, aerothermal, and thermal simulations are combined in order to demonstrate the application of the MDO-U process in TPS design for a HL20 vehicle configuration. Uncertainties in the atmospheric properties and in the thermal properties of the TPS are considered. When uncertainty is accounted in the optimization process, a more conservative optimum is obtained compared to the optimum from the optimization without uncertainty. However, when both optimal configurations are assigned the same total TPS weight through the application of a safety factor on the optimum without uncertainty, the benefits of considering uncertainty during the optimization process are demonstrated through superior performance.

\section{Acknowledgements}

This work was sponsored under Phase I NASA SBIR contract NNA05AC19C. Gary Allen, and David Saunders provided the Traj code and the aerothermal database utilized in this work. Unmeel Mehta was the Technical Monitor for this work. He also identified the HL20 configuration for the optimization study and facilitated the transfer of the necessary technical information for the HL20 from NASA. 


\section{REFERENCES}

1. D.O. Stanley, W.C. England, R.A. Lepsch, M. Mcmillin, K.E. Wurster and R.W. Powell, "Rocket-Powered Single-Stage Vehicle Configuration Selection and Design," AIAA Paper 931053, Feb. 1993.

2. D.O. Stanley, W.C. Engeland, and R.A. Lepsch, "Propulsion System Requirements for Reusable Single-Stage-to-Orbit Rocket Vehicles," AIAA Paper 92-3504, July 1992.

3. P.V. Tartabini, R.A. Lepsch, J.J. Korte K.E. Wurster, "A Multidisciplinary Performance Analysis of a Lifting-Body Single-Stage-to-Orbit Vehicle," AIAA Paper 2000-1045, $38^{\text {th }}$ Aerospace Sciences Meeting \& Exhibit, January 2000, Reno, NV.

4. J. Riehl, C. Trefny, D. Kosareo, "SRM-Assisted Trajectory for the GTX Reference Vehicle," NASA / TM -2000-211599.

5. J.T. Betts, "Using Sparse Nonlinear Programming to Compute Low Thrust Orbit Transfers," The Journal of the Astronautical Sciences, Vol. 41, No. 3, July-September 1993, pp 349-371.

6. J.T. Betts, "Optimal Interplanetary Orbit Transfers by Direct Transcription," The Journal of the Astronautical Sciences, Vol. 42, No. 3, July-September 1994, pp 247-268.

7. P.C. Calhoun and E.M. Queen, "Entry Vehicle Control System Design for the Mars Smart Lander," AIAA-2002-4504, AIAA Atmospheric Flight Mechanics Conference and Exhibit, 5-8 August 2002, Monterey, California.

8. C.E. Cockrell, Jr., "Aerosciences, Aero-Propulsion and Flight Mechanics Technology Development for NASA's Next Generation Launch Technology Program," AIAA 2003-6948, $12^{\text {th }}$ AIAA International Space Planes and Hypersonic Systems and Technologies Conference, December 15-19, 2003, Norfolk, Virginia.

9. C.G. Miller, "Aerothermodynamic Flight Simulation Capabilities for Aerospace Vehicles," AIAA-98-2600, 20 ${ }^{\text {th }}$ AIAA Advance Measurement \& Ground Testing Technology Conference, June 1998, Albuquerque, New Mexico.

10. B.R. Hollis, "Experimental and Computational Aerothermodynamics of a Mars Entry Vehicle," NASA Contractor Report 201633, December 1996.

11. C.G. Miller, "Langley Hypersonic Aerodynamic/Aerothermodynamic Testing Capabilities Present and Future," AIAA Paper 90-1376, 1990.

12. J.A. Dec, J.F. Gasbarre, B.E. George, "Thermal Analysis and Correlation of the Mars Odyssey Spacecraft's Solar Array During Aerobraking Operations," AIAA 2002-4536, AIAA/AAS Astrodynamics Conference, August 2002, Monterey, California.

13. R.M. Amundsen, J.A. Dec, M.C. Lindell, "Thermal Analysis Methods for an Earth Entry Vehicle," Eleventh Thermal and Fluids Analysis Workshop, August 21-25, 2000, Cleveland, Ohio.

14. R.M. Amundsen, J.A. Dec, R.A. Mitcheltree, M.C. Lindell, R.A. Dillman, "Preliminary Thermal Analysis of a Mars Sample Return Earth Entry Vehicle," AIAA 2000-2584, 34 ${ }^{\text {th }}$ AIAA Thermophysics Conference, June 19-22, 2000, Denver, Colorado.

15. TPSX, Thermal Protection System and Material Properties Database, February 1998, NASA Ames Research Center (http://asm.arc.nasa.gov/tpsx).

16. S.J. Scotti, C. Clay, M. Rezin, "Structures and Materials Technologies for Extreme Environments Applied to Reusable Launch Vehicles," AIAA 2003-2697, AIAA/ICAS International Air \& Space Symposium and Exposition, July 14-17, 2003, Dayton, Ohio.

17. M.L. Blosser, "Investigation of Fundamental Modeling and Thermal Performance Issues for a Metallic Thermal Protection System Design," AIAA 2002-0503, $40^{\text {th }}$ Aerospace Sciences Meeting \& Exhibit, January 2000, Reno, Nevada.

18. M.L. Blosser, "Development of Metallic Thermal Protection Systems for the Reusable Launch Vehicle," NASA TM II 0296, October 1996.

19. D.E. Myers, C.J. Martin, M.L. Blosser, "Parametric Weight Comparison of Advanced Metallic, Ceramic Tile, and Ceramic Blanket Thermal Protection Systems," NASA/TM 2000-210289. 
20. R.D. Braun, A.A. Moore, I.M. Kroo, "Use of the Collaborative Optimization Architecture for Launch Vehicle Design," AIAA 1996-1024.

21. S.L. Padula, J.J. Korte, H.J. Dunn, A.O. Salas, "Multidisciplinary Optimization Branch Experience Using iSIGHT Software,” NASA/TM 1999-209714.

22. P.V. Tartabini, R.A. Lepsch, J.J. Korte K.E. Wurster, "A Multidisciplinary Performance Analysis of a Lifting-Body Single-Stage-to-Orbit Vehicle," AIAA Paper 2000-1045, $38^{\text {th }}$ Aerospace Sciences Meeting \& Exhibit, January 2000, Reno, NV.

23. R.D. Braun, and I.M. Kroo, "Development and Application of the Collaborative Optimization Architecture in a Multidisciplinary Design Environment," AIAA 1995-0704.

24. H.M. Kim, "Target Cascading in Optimal System Design," Ph.D. Thesis, University of Michigan, Ann Arbor, 2001.

25. N. Michelena, L. Louca, M. Kokkolaras, C.C. Lin, D. Jung, Z. Filipi, D. Assanis, P. Papalambros, H. Peng, J. Stein, M. Feury, "Design of an Advanced Heavy Tactical Truck: A Target Cascading Case Study," SAE Paper 2001-01-2793.

26. H.M. Kim, M. Kokkolaras, L.S. Louca, G.J. Delagrammatikas, N.F. Michielena, Z.S. Filipi, P.Y. Papalambros, J.L. Stein, and D.N. Assanis, "Target Cascading in Vehicle Redesign: A Class VI Truck Design,” Int. J. of Vehicle Design, vol.29, No.3, 2002, pp.1-27.

27. H.M. Kim, N.F. Michielena, P.Y. Papalambros, I. Jiang, "Target Cascading in Optimal System Design," Transactions of ASME, Journal of Mechanical Design, September 2003, vol.125, pp.474-480.

28. N.F. Michielena, H.A. Park, P.Y. Papalambros, "Convergence Properties of Analytical Target Cascading," Proceedings of the $9^{\text {th }}$ AIAA/ISSMO Symposium On Multidisciplinary Analysis and Optimization, AIAA-2002-5506, Atlanta, GA.

29. Liang, J., Mourelatos, Z. P. and Tu, J., "A Single-Loop Method for Reliability-Based Design Optimization," Proceedings of ASME Design Engineering Technical Conferences (DETC), 2004.

30. Z. P. Mourelatos, N. Vlahopoulos, O. Ebrat, J. Liang, J. Wang, "Probabilistic Main Bearing Performance for an Internal Combustion Engine," ASME Journal of Tribology, v. 127, n 4, October, 2005, p 784-792.

31. G. A. Allen, M. J. Wright, P. Gage, "The Trajectory Program (Traj): Reference Manual and User's Guide," Version 7.2, 20 February 2004, NASA Ames.

32. D. J. Kinney, "Aero-Thermodynamics for Conceptual Design," $42^{\text {nd }}$ AIAA Aerospace Sciences Meeting and Exhibit," 5-6 January 2004, Reno, NV.

33. D.J. Kinney, J.A. Garcia, L. Huynh, "Predicted Convective and Radiative Aerothermodynamic Environments for Various Reentry Vehicles using CBAERO," 44 ${ }^{\text {th }}$ AIAA Aerospace Meeting and Exhibit, 9-12 January 2006, Reno, NV.

34. M.K. McGuire, P. Gage, E.T. Galloway, L. Huyhn, J. Nghyen, J. V. Bowles, R. Windhorst, "Trajectory and Thermal Protection System Design for Reusable Launch Vehicles," $10^{\text {th }}$ AIAA/ISSMO Multidisciplinary Analysis and Optimization Conference, 30 August - 1 September 2004, Albany New York.

35. TPSX, Thermal Protection System and Material Properties Database, February 1998, NASA Ames Research Center (http://asm.arc.nasa.gov/tpsx).

36. M.E. Tauber, K. Sutton, "Stagnation-Point Radiative Heating Relations For Earth And Mars Entries," Journal of Spacecraft and Rockets, vol. 28, No.1, 1991, pp.40-42. 